\title{
A PESSOA IDOSA COM DELIRIUM NA INSTITUCIONALIZAÇÃO
}

\section{Paola Goulart da Rocha ${ }^{1}$; Leris Salete Bonfanti ${ }^{2}$; Naiana Oliveira dos Santos ${ }^{3}$; Maria Helena Gehlen ${ }^{4}$.}

\section{RESUMO}

A pessoa idosa com delirium na institucionalização, apresenta alteração aguda e flutuante do estado mental, redução da consciência e distúrbio na atenção que ocorre em curto ou longo período de tempo. Objetivo: conhecer as potencialidades e fragilidades dos profissionais de saúde ao realizar o rasteio manejo e tratamento da pessoa idosa com delirium na institucionalização.Método: Pesquisa exploratória e descritiva com abordagem qualitativa desenvolvida com entrevista semiestruturada com profissionais de saude de uma Instituição de Longa Permanência para Idosos. Resultados: verificou-se que as potencialiades e fragilidades estão relacionadas e interligadas, principalmente quanto ao surgimento do delirium, em seu contexto multifatorial relacionado a iatrogenia, fatores de risco, existência de uma doença de base e ao ambiente. Conclusão: Ao conhecer as potencialidades e fragilidades considera-se que para rasteio, manejo, tratamento adequado torna-se fundamental - conhecimento da equipe de saúde, acerca dos fatores de risco, critérios para avaliação e principalmente no que tange ao tratamento e a sua prevenção.

Palavras-chave: Idoso; profissionais de saúde; enfermagem geriátrica.

Eixo Temático: Atenção Integral e Promoção á Saúde.

\section{INTRODUÇÃO}

As pessoas idosas constituem 9,2\% da população brasileira, com projeções de que o número de pessoas idosas, até o ano de 2060 , atingirá a faixa de $32,16 \%$

\footnotetext{
${ }^{1}$ Acadêmica de enfermagem, bolsista PROBIC/UFN: Paola Goulart da Rocha - Universidade Franciscana. E-mail: paola.goulart@ufn.edu.br

${ }^{2}$ Professora e coorientadora: Leris Salete Bonfanti Haeffner - Universidade Franciscana. E-mail: lerishaeffner@ufn.edu.br.

${ }^{3}$ Professora colaboradora: Naiana Oliveira dos Santos. - Universidade Franciscana. E-mail: naiana.santos@prof.ufn.edu.br

${ }^{4}$ Professora orientadora: Dra. Maria Helena Gehlen. - Universidade Franciscana. E-mail: mah@ufn.edu.br.
} 
(BRASIL, 2018). Na longevidade, a heterogeneidade entre os idosos é marcante e progressiva durante o processo de envelhecimento (MORAES, 2012). Os processos sindrômicos e as doenças são mais frequentes, e 90\% dos idosos as têm, mas nem sempre estão associadas a dependência funcional (MORAES, 2016).

Dentre esses processos, o delirium é considerado o distúrbio neurológico comportamental com comprovada associação para 0 aumento da morbidade/mortalidade, sendo reconhecido como um problema de saúde pública (BARR et al., 2013; DESSAP et al, 2015; MEHTA et al., 2015).

Diante disto, o processo de envelhecimento individual apresenta estreita associação com o surgimento de agravos transmissíveis como a covid,19, síndromes geriátricas, e de doenças com manifestações complexas como o delirium, que poderá comprometer os sistemas funcionais do idoso e ocasionar dependência de cuidados, fragilização, institucionalização e a hospitalização (MORAES, 2014).

De tal modo, uma pessoa idosa com delirium quando institucionalizada, poderá apresentar uma alteração aguda e flutuante do estado mental, com redução da consciência e distúrbio da atenção, que não pode ser atribuída a uma demência previamente existente ou em evolução, pois ocorre em curto período de tempo, geralmente de horas a dias, com tendência a flutuar ao longo do dia (BISINOTTO et al., 2017).

Tendo em vista a problemática apresentada, questiona-se: Quais as potencialidades e fragilidades dos profissionais de saúde ao realizarem o rasteio, manejo e tratamento da pessoa idosa com delirium na institucionalização? E, objetiva-se conhecer as potencialidades e fragilidades dos profissionais de saúde ao realizar o rasteio manejo e tratamento da pessoa idosa com delirium na institucionalização.

\section{METODOLOGIA}

Pesquisa exploratória e descritiva com abordagem qualitativa, que em sua abrangência, considera na realidade investigada, comportamentos, historicidade, relações, representações, crenças, percepções, opiniões, interpretações, atitudes e 
posturas das pessoas que a constituem, bem como as configurações dos aspectos organizacionais, do seu cotidiano, do que sentem e do que pensam (BARDIN, 2016).

Este estudo é pertencente ao atendimento do objetivo secundário do projeto de pesquisa de iniciação científica PROBIC/UFN, denominado: Rasteio, manejo e tratamento da pessoa idosa com delirium na institucionalização aprovado pelo Comitê de Ética e Pesquisa (CEP) da UFN, com o número 40736420.3.0000.5306 sendo que está pesquisa permenece em vigência até o ano de 2022.

De tal modo, foi realizada uma entrevista semiestruturada (MARCONI; LAKATOS, 2003), com sete profisssionais de saúde, de uma Instituição de Longa Permanência para Idosos (ILPI). Elegeu-se como critérios de inclusão: ser profissional de saúde com atuação de três meses. Optou-se pela utilização do período de três meses, por ser o tempo de estágio probatório do cargo. Foram excluídos os profissionais de saúde que estavam afastados de suas atividades laborais, no período da coleta de dados, por motivo de natureza particular.

A Coleta de dados, foi realizada após o esquema vacinal da covid 19, dos participantes da pesquisa. Utilizou-se a Análise do Conteúdo de Bardin (2016), a qual consiste em um conjunto de técnicas de análise das comunicações visando obter, por procedimentos sistemáticos e objetivos de descrição do conteúdo das mensagens, indicadores que permitam a inferência de conhecimentos relativos às condições de produção e de recepção dessas mensagens.

Segundo a autora, é um método de tratamento da informação contida nas mensagens que possibilita a criação de categorias relacionadas ao objeto de pesquisa. A operacionalização da análise contemplou, três momentos: pré-análise, exploração do material, interpretação e tratamento dos resultados obtidos, para conhecer as potencialidades e fragilidades dos profissionais de saúde ao realizarem o rasteio, manejo, e tratamento da pessoa idosa com delirium na institucionalização .

\section{RESULTADOS E DISCUSSÕES}

Verificou-se, que uma das estratégias que configuram a potencialidade, se relacionou com o conhecimento dos profissionais de saúde, de que a pessoa idosa 
com delirium na institucionalização, necesssita receber atendimento de suas necessidades humanas básicas de vida identificando o delirium precocemente.

A ILPI tem entre suas funções promover o bem-estar, manutenção da funcionalidade, e melhoria da qualidade de vida no cotidiano, com monitoramento constante que permita auxiliar no melhor manejo clínico funcional do idoso (PESSOA, et.al., 2019). Nesse contexto, reconhece-se que os profissionais de saúde assumem importante papel, no rasteio, manejo e tratamento da pessoa idosa com delirium na ILPI, em garantia a manutenção da sua funcionalidade, prevenção da hospitalização principalmente durante a pandemia de covid 19 e melhoria do seu bem-estar.

A partir da pré análise do material, identificou-se como possibilidade de efetividade do rasteio, manejo e tratamento da pessoa idosa com delirium na institucionalização, a especialização do profissional de saúde para o atendimento geriátrico e gerontológico.

No momento da exploração dos achados investigados, para conhecer o rasteio, manejo e tratamento da pessoa idosa com delirium na institucionalização, realizado pelos participantes da pesquisa, foi possivel caracterizar os profissionais a partir, da sua atuação profissional, conforme as características, apresentadas no quadro 1

Quadro 1: Descrição das caracteristicas da atuação profissional

\begin{tabular}{|l|l|}
\hline Idade do participante: & Entre 23 e 48 anos \\
\hline Categoria profissional: & Enfermeiros \\
\hline $\begin{array}{l}\text { Especializações } \\
\text { profissionais: }\end{array}$ & $\begin{array}{l}\text { três participantes com especialização em: gestão em enfermagem, } \\
\text { urgência e emergência, enfermagem do trabalho e sistema público de } \\
\text { saúde. }\end{array}$ \\
\hline $\begin{array}{l}\text { Tempo de serviço na } \\
\text { ILPI: }\end{array}$ & O tempo varia entre três meses á nove anos. \\
\hline
\end{tabular}

Quadro 1: elaborado pela autora.

De tal modo, interprata-se de acordo com Moraes (2014), que o processo de envelhecimento individual apresenta estreita associação com o surgimento de agravos e manifestações complexas como o delirium, que poderá comprometer os sistemas funcionais do idoso e ocasionar dependência de cuidados, fragilização. $O$ 
que poderá ocasionar na pessoa idosa que esta institucionalida a hospitalização.

Assim percebe-se que a possibilidade de efetividade do rasteio, manejo e tratamento da pessoa idosa com delirium na institucionalização, a especialização do profissional de saúde, para o atendimento geriátrico e gerontológico como uma fragilidade, porém com viabilidade de transformação desta realidade, de que os participantes posssuem especilização não direcionada a atuação profissional no atendimento da população idosa.

Apesar da notável incidência do delirium no idoso institucionalizado, estudo comprovou que profissionais de saúde apresentam conhecimento limitado sobre este tema, desconsiderando a gravidade do problema (CHRISTENSEN, 2014). A condição de saúde do idoso com delirium é relacionada à promoção da sua vitalidade com independência na institucionalização, rastreio precoce da vulnerabilidade, pois quanto maior a dependência de cuidados, maior é o risco e a chance de fragilidade e hospitalização (NUNES; MENEZES; ALCHIERI, 2010).

Quanto a compreensão dos participantes da pesquisa, sobre a potencialidade de identificar precocemente o delirium foi averiguado que sete participantes demostraram sua compreensão a cerca do delirium, com respostas variadas entre: Perturbação Mental; alteração do estado mental em tempo e espaço; alteração da capacidade cognitiva; confusão mental. Além disso, a fragilidade foi considerada perante o conhecimento adquirido somente durante a graduação, sendo que ao vivenciarem o delirium na ILPI, alegarm ser um desafio com um certo grau de dificuldade e tranquilidade.

O delirium é multifatorial relacionado a iatrogenia, fatores de risco, existência de uma doença de base e ao ambiente (MORAES et al., 2019). Os fatores de risco são divididos, de acordo com sua predisposição, sendo considerados não modificáveis e modificáveis (BISNOTTO et al., 2017).

São descritos como fatores de risco modificáveis, o ambiente, ruídos sonoros e iluminação artificial, mudança de hábitos, privação do sono, isolamento, o rodízio de profissionais, contenção física, dor, presença de procedimentos invasivos como tubos, sondas, ventilador mecânico, administração de medicamentos devido à dor aguda, entre outros. Entre os fatores não modificáveis, destacam-se a idade 
avançada, hábitos pessoais, história de tabagismo, comorbidades, doenças prévias do sistema nervoso e características genéticas (PITROWSKY et al., 2010).

\section{CONCLUSÃO}

Considera-se que ao conhecer as potencialiades e fragilidades dos profissionais de saúde ao realizar o rasteio, manejo e tratamento da pessoa idosa com delirium na institucionalização é imprescindível, relacionar com a necessidade de conhecimento especializado da equipe de saúde, acerca dos fatores de risco, critérios para avaliação e principalmente, no que tange ao tratamento e a sua prevenção.

\section{AGRADECIMENTOS}

A Universidade Franciscana pela bolsa concedida de IC PROBIC/UFN; o evento pela oportunidade de apresentar o nosso trabalho; as colegas de profissão pela participação.

\section{REFERÊNCIAS}

BARR, J.et al. Clinical Practice Guidelines for the Management of Pain, Agitation, and Delirium in Adult Patients in the IntensiveCare Unit. CriticalCare Medicine, [s.I.], v.41, n.1, p.263-306, jan. $2013 . \quad$ Doi: http://dx.doi.org/10.1097/ccm.0b013e3182783b72.

BARDIN, L. Análise de conteúdo. 3. ed. Lisboa, 2016.

BISNOTTO, F.M.B. et al. Delirium pós-operatório no idoso "Onde estamos?" RevMed Minas Gerais. 2017; v. 27, n 2, p. 52-66.

CAMARANO, A.A.; KANSO, S. As instituições de longa permanência para idosos no Brasil. Rev. bras. estud. popul. v.27, n.1, P.233-235, 2010.

CHRISTENSEN, Mn. Anexploratorystudyof staff nurses' knowledgeof delirium in the medical ICU: AnAsian perspective. Intensive And CriticalCareNursing, [s.I.], v. 30, n. 1, p.54-60, fev. 2014. Elsevier BV. Doi: http://dx.doi.org/10.1016/i.iccn.2013.08.004. 
DESSAP, A.M. et al. Delirium and circadian rhythm of melatonin during waning from mechanical ventilation: an ancillary study of wearing trial. Chest. v.148, n.5, p. 1231 41. 2015.

HAGUETTE, T. M. F. Metodologias qualitativas na sociologia. 3. ed. PetrópolisRJ: Vozes, 1992.

LENARDT, M.H; MICHEL, T; TALLMANN, A.E.C. A condição de saúde de idosas residentes em instituição de longa permanência. Cogitare Enferm, v. 14, n. 2, p. 227-36, 2009.

MARCONI, M. A.; LAKATOS, E. M. Fundamentos da Metodologia Científica. 5. ed. São Paulo: Atlas S.A, 2003.

MEHTA, S. et al. Prevalence, risk factors, and outcomes of delirium in mechanically ventilated adults. Crit Care Med. v. 43, n.3, p.557-66. 2015.

MORAES, E. N. Atenção à saúde do Idoso: Aspectos Conceituais. Brasília: Organização Pan-Americana da Saúde, 2012.

- Como preparar o sistema de saúde brasileiro para enfrentar o envelhecimento, tendo em vista a mudança do perfil demográfico. Revista Consensus, v. 10, p. 40-45, 2014.

Princípios básicos em Geriatria e Gerontologia. Coopmed, 2009.

MORAES, E. N.\& MORAES, F. L. Coleção Guia de Bolso em Geriatria e Gerontologia: Avaliação multidimensional do Idoso. 5. ed. Belo Horizonte: Folium, 2016.

MORAES JUNIOR, R.F. et al. Principais fatores de risco para delirium encontrados nos idosos internados nas enfermarias de clínica médica de um hospital da Amazônia. REAS/EJCH. v.17, n.e272, p.1-8. 2019. DOI: https://doi.org/10.25248/reas.e272.2019

PESSOA, L.S.C. et al. Cuidado de enfermagem ao idoso com delirium em unidade intensiva. Ver enferm UFPE online. v.13, p.1-7. 2019. Doi: https://doi.org/10.5205/1981-8963.2019. 239682

PITROWSKY, M. T. et al. Importância da monitorização do delirium na unidade de terapia intensiva. RevBras Ter Intensiva, [s.i.], v.3, n.22, p.274-279. 2010. 
EDUCAÇÃO, SAÚDE

ETECNOLOCIA

26 A 28 DE OUTUBRO DE 2021

\section{QUFN}

Disponível em: <http://www.scielo.br/pdf/rbti/v22n3/10.pdf>. Acesso em: 29 set. 2018. 